\title{
ESTUDO COMPARATIVO ENTRE BLINDAGENS MULTICAMADA UTILIZANDO COMPÓSITOS POLIÉSTER-SISAL*
}

Lucas Tedesco Bolzan' Fábio de Oliveira Braga² Sérgio Neves Monteiro ${ }^{3}$

\section{Resumo}

Blindagens contra armas de fogo são de grande importância para proteção do homem, no contexto atual de violência urbana e conflitos armados. Destacam-se os sistemas de blindagem multicamada (SBMs), constituídos por materiais diferentes, pelo seu bom desempenho e menor peso. Um SBM típico é constituído de uma camada cerâmica frontal, uma segunda camada de compósito e uma camada posterior de metal dúctil. Apesar de serem tipicamente fabricados em materiais sintéticos, uma alternativa é a utilização de compósitos reforçados com fibras naturais como segunda camada. Este trabalho tem como objetivo verificar a eficiência de SBMs que utilizam compósitos de poliéster com $30 \%$ vol. de fibras ou tecido de sisal. Foram realizados ensaios balísticos com munição 7,62 mm, e verificou-se que os SBMs com fibras e tecido de sisal se enquadram no critério da norma internacional (NIJ 0101.06), de resistir ao impacto balístico e imprimir deformação menor que $44 \mathrm{~mm}$ no material de referência (plastilina), além de manterem sua integridade após o impacto.

Palavras-chave:Blindagem multicamada; Impacto balístico; Fibra de sisal; Compósito de poliéster.

\section{COMPARATIVE STUDY BETWEEN MULTILAYERED ARMORS USING POLYESTER-SISAL COMPOSITES}

\section{Abstract}

Armor against firearms has great importance to personal protection of man, in the present context of urban violence and armed conflicts. Multilayered armor systems (MAS), consisting of different materials, stand out due to their good performance and less weight. A typical MAS is made of a frontal ceramic layer, a composite as second layer and a ductile metal back layer. Despite of being typically made using synthetic materials, an alternative is using natural fiber reinforced composites as second layer. This work aims to verify the efficiency of MAS that use polyester composites reinforced with 30 vol.\% sisal fibers or woven fabrics. Ballistic tests were carried out with $7.62 \mathrm{~mm}$ ammunition, and it has been verified that MAS with sisal fibers and woven fabric fit the criteria of the international standard (NIJ 0101.06), of resisting to the ballistic impact and to cause deformation less than $44 \mathrm{~mm}$ in the reference material (modelling clay), besides keeping the layer integrity after the impact.

Keywords: Multilayered armor; Ballistic impact; Sisal fiber; Polyester composite.

1 Engenheiro Civil, mestrando em Ciência dos Materiais, Seção de Engenharia Mecânica e de Materiais, Instituto Militar de Engenharia (IME), Rio de Janeiro, RJ, Brasil

2 Engenheiro Metalurgista, M.Sc., Doutorando em Ciência dos Materiais, Seção de Engenharia Mecânica e de Materiais, Instituto Militar de Engenharia, Rio de Janeiro, RJ, Brasil. fabio_obraga@yahoo.com.br.

3 Engenheiro Metalurgista, Ph.D, Professor Titular, Seção de Engenharia Mecânica e de Materiais, Instituto Militar de Engenharia, Rio de Janeiro, RJ, Brasil. 


\section{INTRODUÇÃO}

A tecnologia da blindagem é fundamental, seja na guerra moderna ou nas guerrilhas urbanas que se alastram pelas diversas cidades do mundo todo ${ }^{1}$. Atualmente armas letais, como fuzis de calibre 7,62 mm, por exemplo, são de fácil acesso para traficantes e isso constitui sério risco a segurança urbana.

O desenvolvimento nos processos tecnológicos vem acarretando armamentos cada vez mais potentes e com alto poder destrutivo, no entanto, contribui também com correspondentes sistemas de proteção balística. É necessário aumentar a eficiência das blindagens, visto que as ameaças com armamentos modernos são crescentes ${ }^{2}$. A busca por estruturas de proteção é interminável, uma vez que está associada com ameaças que colocam em risco vidas humanas ${ }^{3}$.

A composição das blindagens até a Segunda Guerra Mundial consistia basicamente em metais, porém, as blindagens começaram a ficar grandes e pesadas à medida que novos projéteis iam sendo desenvolvidos. Como alternativa, materiais leves e com maior desempenho começaram a ser utilizados ${ }^{4}$.

Atualmente, já se sabe que um único material não consegue conter as tensões mecânicas que resultam do impacto de um projétil calibre $7,62 \mathrm{~mm}$ ou superior, exceto em casos de grandes espessuras, que se mostram inadequados para uso pessoal. Isso explica a utilização de mais de uma camada de proteção nas chamadas blindagens multicamada, associando diferentes materiais, como cerâmicas, polímeros, compósitos reforçados com fibras e metais.

Para a primeira camada geralmente se escolhe um material cerâmico, que ao receber o impacto inicial vai erodir e fraturar a ponta do projétili, ${ }^{5,6}$, dissipando energia cinética de impacto através da fragmentação ${ }^{7}$, que envolve nucleação, crescimento e coalescência de microfissuras ${ }^{8}$.

A segunda camada pode fazer uso de materiais compósitos ou poliméricos, como poliéster, poliamida, aramida, polietileno e polipropileno ${ }^{9}$. Ela possui a função de absorver energia proveniente dos fragmentos do projétil e da cerâmica ${ }^{10}$. Dentre os materiais mais utilizados convencionalmente destaca-se o laminado fabricado a partir das fibras de aramida (comercialmente conhecido como Kevlar ${ }^{\circledR}$, Twaron® ou Gold Shield®, por exemplo), ou de polietileno de ultra-alto peso molecular (conhecido como Dyneema ${ }^{\circledR}$ ou Spectra®. Recentemente verificou-se que fibras naturais em compósitos poliméricos também são efetivas ${ }^{10}$.

As fibras naturais lignocelulósicas (FNLs), que desde a última década vem sendo avaliadas em artigos científicos, tem se tornado interessantes na fabricação de compósitos para fins de blindagem balística. Seu uso vem ganhando destaque, visto que elas são baratas, biodegradáveis, possuem baixa densidade, não são abrasivas e suas propriedades são comparáveis às outras fibras utilizadas como reforço ${ }^{11}$. Com relação às questões ambientais, seu descarte não gera problemas e elas não são tóxicas ${ }^{12}$, além do fato de serem neutras com relação ao dióxido de carbono $(\mathrm{CO} 2)^{13}$.

A substituição de materiais sintéticos (como aramida) por naturais reduz o gasto energético, emissões de gases poluentes, custos associados aos processos de fabricação e gera reaproveitamento de material. A aplicação das FNLs em sistemas de blindagem balística, além de promover todas as vantagens citadas anteriormente pode suprir necessidades do militarismo, possibilitando a produção de blindagens leves e eficientes, contribuindo com a segurança nacional e os avanços da pesquisa científica. 


\section{MATERIAIS E MÉTODOS}

Neste trabalho, serão avaliados sistemas de blindagem multicamada compostos por três camadas: uma cerâmica, um compósito polimérico reforçado com fibras naturais e uma chapa de alumínio.

A cerâmica frontal possui formato de um hexágono com $10 \mathrm{~mm}$ de espessura, é composta por alumina $\left(\mathrm{Al}_{2} \mathrm{O}_{3}\right)$ dopada com $4 \%$ em peso de nióbia $\left(\mathrm{Nb}_{2} \mathrm{O}_{5}\right)$ e foi sinterizada a $1400{ }^{\circ} \mathrm{C}$ por aproximadamente 3 horas, no Laboratório de Materiais Cerâmicos do Instituto Militar de Engenharia (IME), na cidade Rio de Janeiro (RJ), Brasil.

Para o compósito da camada intermediária utilizou-se uma matriz de poliéster reforçada com $30 \%$ em volume de sisal, disposto na forma de fibras, alinhadas em uma única direção, e tecido. Essa camada é retangular nas dimensões $120 \times 150$ $\mathrm{mm}$, com $10 \mathrm{~mm}$ de espessura. As fibras foram fornecidas pela empresa Eletro Ferragens Santa Domênica, em pacotes de $500 \mathrm{~g}$, e o tecido foi adquirido por meio da empresa Sisalsul - Fibras Naturais. O sisal foi limpo com auxílio de uma escova, e levado à estufa a 60 $\mathrm{C}$, onde permaneceu por 24 horas. Em seguida foi disposto em uma matriz metálica, onde também foi colocada a resina de poliéster, já misturada com um endurecedor, a fim de promover a cura do material. Sobre o molde metálico foi aplicada uma pressão de 5 toneladas com auxílio de uma prensa hidráulica. Após 24 horas as amostras foram retiradas do molde, pesadas e medidas. A Figura 1 ilustra: (a) uma camada de cerâmica; (b) uma placa de compósito reforçado com fibras de sisal; (c) uma placa de compósito reforçado com tecido de sisal.

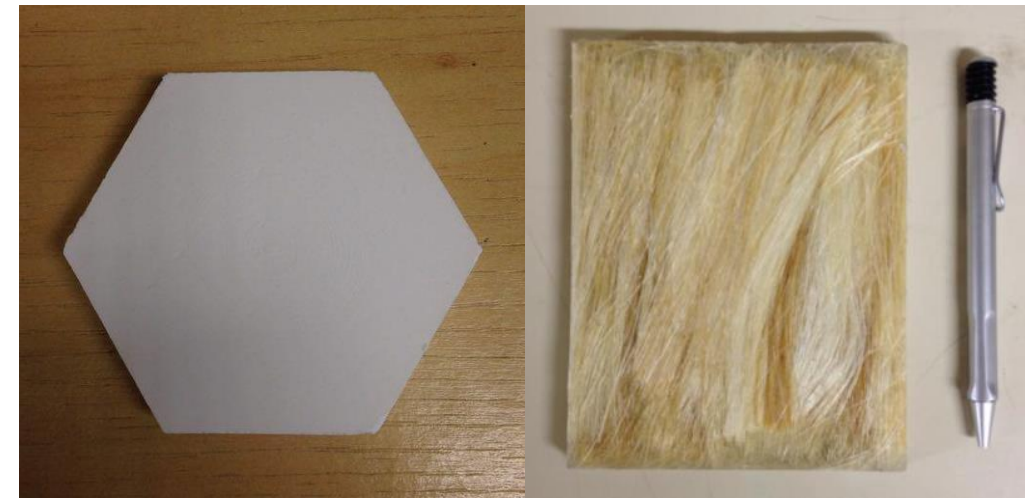

(a) (b)

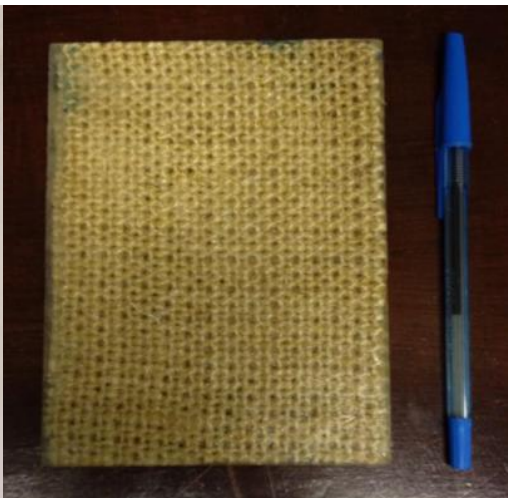

(c)

Figura 1. (a) Camada frontal de cerâmica; (b) Camada intermediária de poliéster reforçado com fibras de sisal; (c) Camada intermediária de poliéster reforçado com tecido de sisal.

A última camada do sistema de blindagem consiste em uma fina chapa fabricada com liga de alumínio 5052-H34 no formato retangular, com dimensões $120 \times 150$ $\mathrm{mm}$, com $5 \mathrm{~mm}$ de espessura. A colagem das três camadas foi realizada com adesivo de cura rápida ULTRAFLEX, a base de poliuretano. A Figura 2 ilustra os sistemas de blindagem multicamada depois de prontos. 


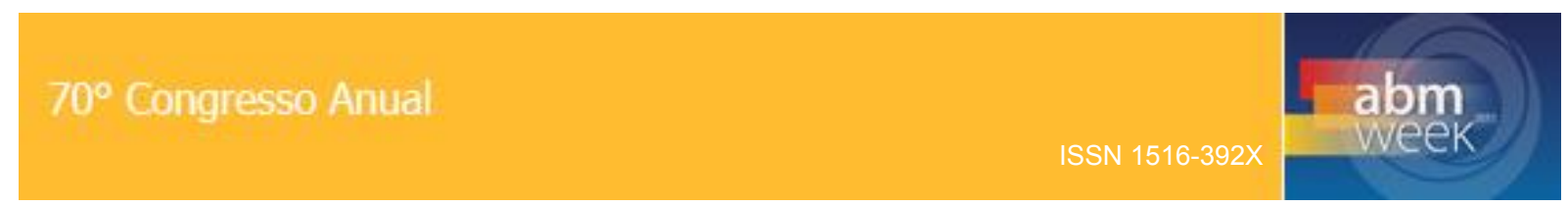

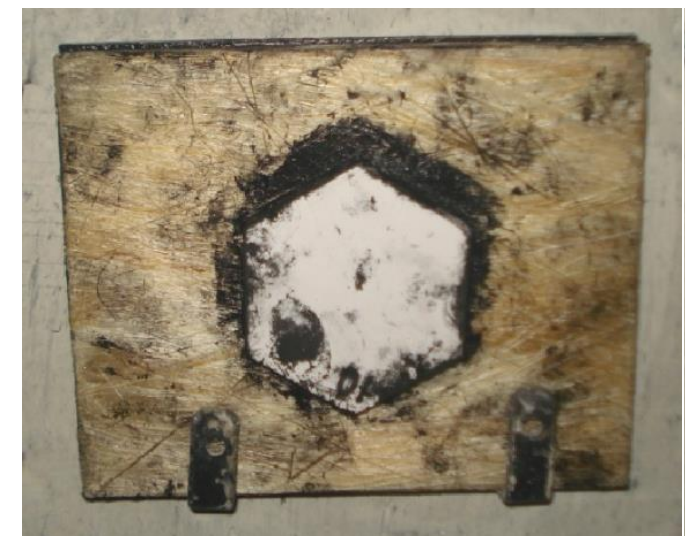

(a)

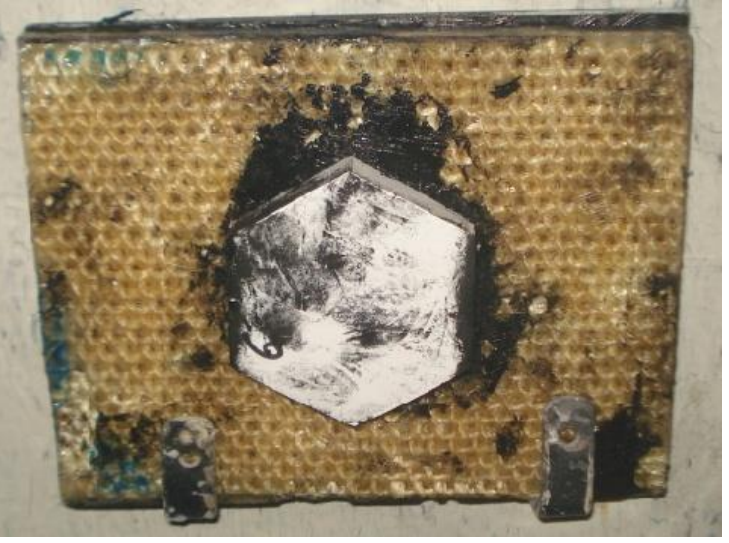

(b)

Figura 2. Amostras do sistema de blindagem multicamada com compósito de poliéster reforçado com $30 \%$ de sisal, no momento anterior ao impacto balístico: (a) Sisal na forma de fibras; (b) Sisal na forma de tecido.

O nível de proteção dos sistemas de blindagem multicamada foi avaliado com base nos resultados dos ensaios balísticos, de acordo com a norma NIJ 0101.06 (2008).Os testes foram executados no Centro de Avaliações do Exército (CAEx), no Campo de Prova da Marambaia, Rio de Janeiro. O ensaio balístico consistiu no disparo de um projétil $7,62 \mathrm{~mm}$ contra o alvo (material a ser avaliado), posicionado frente a um bloco de plastilina (CORFIX®) de $50 \mathrm{~mm}$ e massa específica de 1,7 $\mathrm{g} / \mathrm{cm}^{3}$, que simula a consistência do corpo humano. Foi então medida da profundidade de deformação provocada pelo tiro. A distância de disparo (da boca da arma até o ponto de contato com o material a ser atingido) foi de 15 metros. As velocidades de impacto do projétil no alvo foram registradas com auxílio do radar Doppler, modelo SL-520P, do fabricante Weibel. Para avaliar os resultados obtidos nos ensaios foi utilizado o método estatístico de Weibull.

\section{RESULTADOS E DISCUSSÃO}

Em todos os ensaios balísticos o projétil foi parado pelos sistemas de blindagem multicamada, indicando dissipação de energia cinética pelo sistema. A energia residual está diretamente associada com o trauma que provocou a deformação no bloco de massa, que pode ser visualizado na Figura 3.

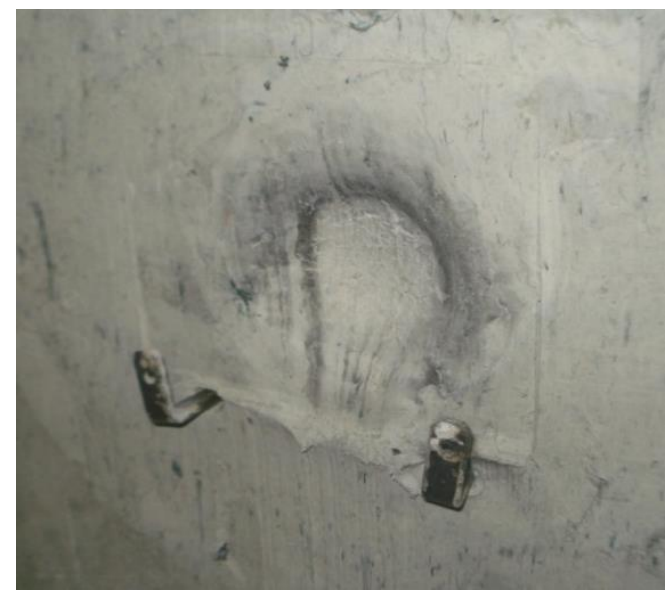

Figura 3. Deformação causada no bloco de massa, que resultou do impacto balístico. 
Estão apresentados na Tabela 1 os valores médios de profundidade no bloco de massa, velocidade $\left(V_{0}\right)$ e energia de impacto $\left(E_{0}\right)$ do projétil, bem como as características físicas (espessura e densidade) das amostras de poliéster-fibra de sisal (P-30\% FS) e de poliéster-tecido de sisal (P-30\% TS).

Tabela 1. Valores médios obtidos na caracterização das camadas intermediárias das blindagens multicamada e nos ensaios balísticos.

\begin{tabular}{cccccc}
$\begin{array}{c}\text { Camada } \\
\text { intermediária }\end{array}$ & $\begin{array}{c}\text { Espessura } \\
(\mathbf{m m})\end{array}$ & $\begin{array}{c}\boldsymbol{\rho} \\
\left(\mathbf{g} / \mathbf{c m}^{\mathbf{3}}\right)\end{array}$ & $\begin{array}{c}\text { Profundidade } \\
(\mathbf{m m})\end{array}$ & $\boldsymbol{V}_{\mathbf{0}}(\mathbf{m} / \mathbf{s})$ & $\boldsymbol{E}_{\mathbf{0}}(\mathbf{K J})$ \\
\hline P-30\% TS & 11,05 & 1,17 & $24,3 \pm 3,3$ & 837,51 & 3,40 \\
P-30\% FS & 11,09 & 1,16 & $21,7 \pm 3,1$ & 841,03 & 3,43 \\
\hline
\end{tabular}

Analisando a Tabela 1, é possível notar que os dois tipos de amostras apresentaram profundidade média de deformação no bloco de massa inferior a $44 \mathrm{~mm}$, que é o valor máximo especificado na norma NIJ 0101.06 (2008) para o nível III (7,62 mm), portanto, ambos os sistemas de blindagem multicamada se mostraram eficientes. As amostras de poliéster reforçado com tecido de sisal apresentaram profundidade média de deformação de 24,3 $\pm 3,3 \mathrm{~mm}$, ou seja, se mostraram igualmente eficientes às de poliéster reforçado com fibras de sisal, que obtiveram $21,7 \pm 3,1$ $\mathrm{mm}$. Devido à proximidade nos valores, pode-se supor que as fibras isoladas se distribuíram de forma igualmente uniforme que os tecidos no interior da matriz metálica, resultando em um mesmo grau de coesão e consequentemente resistência mecânica do compósito. Isso explica o desempenho balístico semelhante dos diferentes tipos de amostras.

A delaminação parece ser um dos principais mecanismos de fratura das amostras de poliéster reforçadas com sisal na forma de fibras, sendo percebida a olho nu (indicada por setas na Figura 4). Ela é o resultado da separação das fases do compósito, e juntamente com outros mecanismos, como a fratura frágil da matriz polimérica, contribui para dissipação de energia cinética. É conhecido que a resistência balística do material está diretamente associada a dois processos principais: a absorção de energia e a redistribuição da energia do projétil ${ }^{2}$. A delaminação, como mecanismo de fratura, pode promover ambos os processos, pois absorve energia através de fratura, e redistribui a energia para regiões afastadas do impacto (Figura 4). 


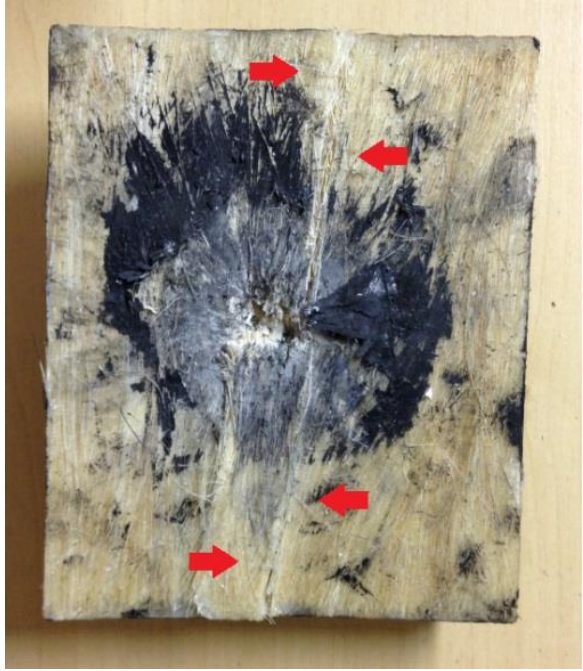

Figura 4. Amostra de poliéster reforçada com 30\% de fibras de sisal, após sofrer impacto balístico. As setas indicam o mecanismo de delaminação.

É importante destacar que todas as amostras de poliéster-sisal se mantiveram íntegras após o impacto balístico. Esta característica é interessante para blindagens pessoais: se um primeiro disparo não é capaz de fragmentar a blindagem, significa que ela continua sendo eficiente para uma segunda tentativa, oferecendo uma maior proteção ao usuário.

Os resultados dos ensaios balísticos foram relacionados através do método estatístico de Weibull. A Figura 5 ilustra os gráficos da distribuição para as amostras com fibras e tecido de sisal. A Tabela 2 fornece os parâmetros de Weibull.
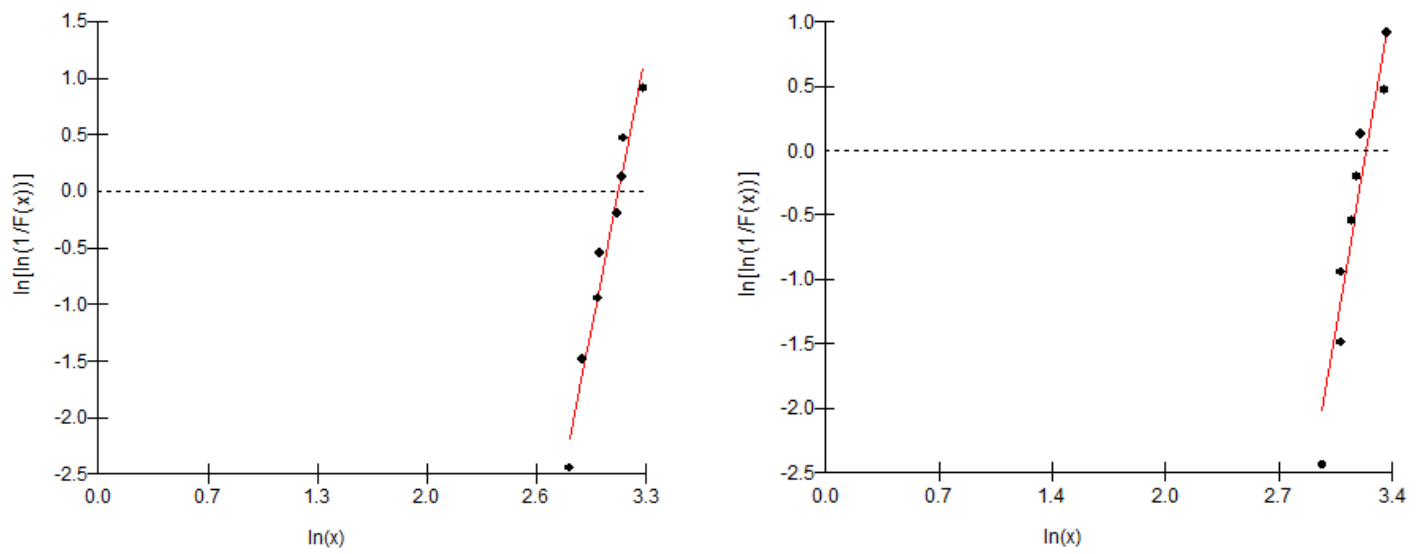

(a)

(b)

Figura 5. Gráficos da distribuição de Weibull das profundidades de deformação para as blindagens com compósitos de poliéster reforçado com 30\% de:

(a) Fibras de sisal; (b) Tecido de sisal. 
Tabela 2. Parâmetros de Weibull dos resultados balísticos das blindagens multicamada com compósitos poliéster-sisal

\begin{tabular}{cccc}
$\begin{array}{c}\text { Camada } \\
\text { intermediária }\end{array}$ & $\begin{array}{c}\text { Módulo de } \\
\text { Weibull }(\boldsymbol{\beta})\end{array}$ & $\begin{array}{c}\text { Unidade } \\
\text { característica } \\
(\boldsymbol{\theta})\end{array}$ & $\begin{array}{c}\text { Coeficiente } \\
\text { de correlação } \\
\left(\boldsymbol{R}^{2}\right)\end{array}$ \\
\hline 30\% Tecido & 7,653 & 25,64 & 0,9222 \\
$30 \%$ Fibras & 7,357 & 23,00 & 0,9642 \\
\hline
\end{tabular}

Nas duas situações, os pontos correspondentes às profundidades de deformação apresentaram comportamento unimodal, com os dados bem ajustados à reta de Weibull. É possível verificar na Tabela 2 que foram obtidos excelentes ajustes, pois o $\mathrm{R}^{2}$ se mostrou superior a 0,9 em ambos os casos. Altos valores de $\mathrm{R}^{2}$ indicam que a dispersão dos valores da profundidade de deformação tem origem em fenômenos aleatórios, e assim, com $\mathrm{R}^{2}>0,90$, pode-se afirmar que mais de $90 \%$ da variação da profundidade pode ser explicada por fenômenos aleatórios, com 95\% de confiança. Se houvesse algum fenômeno não-aleatório agindo, o gráfico apresentaria comportamento bimodal, ou de ordem superior, e o valor de $\mathrm{R}^{2}$ seria baixo, pois os dados não se ajustariam à reta. Além disso, o parâmetro $\beta$ é uma medida de quão estreita é a distribuição, ou seja, quanto maior $\beta$, mais homogêneos e próximos da média são os dados. O compósito reforçado com $30 \%$ de tecido de sisal apresentou a maior homogeneidade dos dados $(\beta=7,653)$, no entanto, maior valor de $\theta(25,64)$, que nesse caso representa a profundidade de deformação característica. Entretanto, em geral, considera-se o comportamento balístico do poliéster reforçado fibras e tecido de sisal com desempenho similar.

\section{CONCLUSÃO}

- $\quad$ Os sistemas de blindagem multicamada analisados no presente trabalho são considerados eficientes com relação ao critério adotado pela norma NIJ 0101.06 (2008), ou seja, a profundidade de deformação causada no bloco de massa após o impacto balístico não excedeu o máximo permitido $(44 \mathrm{~mm})$, independente da camada intermediária utilizada (compósito de poliéster reforçado com fibras ou tecido de sisal).

- $\quad$ O sistema de blindagem multicamada que utiliza como camada intermediária o compósito de poliéster reforçado com fibras de sisal se mostrou igualmente efetivo ao sistema equivalente que utiliza tecido de sisal.

- $\quad$ Com relação ao sistema de blindagem multicamada que possui como camada intermediária o compósito de poliéster reforçado com fibras de sisal, a delaminação se mostrou como um dos principais mecanismos de fratura.

- Todas as camadas intermediárias de poliéster reforçado com sisal se mantiveram íntegras após o impacto balístico, independentemente se o sisal estava na forma de fibras ou tecido. Isso é uma grande vantagem para sistemas de blindagem balística. 


\section{Agradecimentos}

Os autores deste trabalho agradecem às agências brasileiras CNPq e CAPES, pelo apoio financeiro, e também ao CAEx, por disponibilizar o local de realização dos ensaios balísticos.

\section{REFERÊNCIAS}

1 Junior WFA, Santos MA, Bastian FL, Suarez JCM, Cardoso AL, Chaves QG.

Comportamento sob impacto balístico de um sistema compósito para blindagem.

Revista Eletrônica de Materiais e Processos. 2006;1:12-16.

2 Wang L, Kanesalingam S, Nayak R, PadhyeR. Recent trends in Ballistic Protection. Textiles and Light Industrial Science and Technology. 2014;3:37-47.

3 Serjouei A, Chi R, Zhang Z, Sridharl. Experimental Validation of BLV Model on Bi-Layer Ceramic-Metal Armor. International Journal of Impact Engineering. 2015;77:30-41.

4 Bürger D, De Faria AR, De Almeida SF, De Melo FC, Donadon MV. Ballistic impact simulation of an armour-piercing projectile on hybrid ceramic/fiber reinforced composite armours. International Journal of Impact Engineering. 2012;43:63-77.

5 Da Silva MV, Stainer D, Al-Qureshi HA, Hotza D. Blindagens Cerâmicas para Aplicações Balísticas: Uma Revisão. Cerâmica. 2014; 60:323-331.

6 Shokrieh MM, Javadpour GH. Penetration analysis of a projectile in ceramic composite armor. Composite Structures. 2008; 82:269-276.

7 Medvedovski E. Ballistic Performance of Armour Ceramics: Influence of Design and Structure - Part 1. Ceramics International. 2010; 36:2103-2115.

8 Louro LHL, Meyers MA. Effect of stress state and microstructural parameters on impact damage of alumina-based ceramics. Journal of materials science. 1989; 24:2516-2532.

9 Lopes MA,Gonçalves DP, De Melo F. Resistência balística de compósitos poliméricos laminados [Anais do9 Congresso Brasileiro de Polímeros;2007 Out. 7-11; Campina Grande, Brasil].

10 Monteiro SN, Louro LHL, Trindade W, EliasCN, Ferreira CL, Lima ES, Weber RP, Suarez JCM, Figueiredo ABS, Pinheiro WA, Silva LC, Lima Jr EP. Natural Curaua FiberReinforced Composite in Multilayered Ballistic Armor.Metallurgical and Materials Transactions A. 2015;46:4567-4577.

11 Nabi Saheb D, Jog JP. Natural Fiber Polymer composites a review. Advances in Polymer Technology. 1999; 18:351-363.

12 Carvalho LH, Cavalcanti WS. Propriedades Mecânicas de Tração de Compósitos Poliéster/Tecidos Híbridos Sisal/Vidro. Polímeros. 2006;16: 33-37.

13 Crocker J. Natural materials innovative natural composites. Materials Technology. 2008;23:174-178. 